\title{
Student Monitoring System using Machine Learning
}

\author{
Ashok Kumar S, Ragul R N, Gokula krishnan D, Praveen Kumar S
}

\begin{abstract}
The period behavioral engagement is commonly used to describe the scholar's willingness to participate within the getting to know the system. Emotional engagement describes a scholar's emotional attitude toward learning. Cognitive engagement is a chief part of overall learning engagement. From the facial expressions the involvement of the students in the magnificence can be decided. Commonly in a lecture room it's far difficult to recognize whether the students can understand the lecture or no longer. So that you can know that the comments form will be collected manually from the students. However the feedback given by the students will now not be correct. Hence they will no longer get proper comments. This hassle can be solved through the use of facial expression detection. From the facial expression the emotion of the students may be analyzed. Quantitative observations are achieved in the lecture room wherein the emotion of students might be recorded and statistically analyzed. With the aid of the use of facial expression we will directly get correct information approximately college students understand potential, and determining if the lecture becomes exciting, boring, or mild for the students. And the apprehend capability of the scholar is recognized by the facial emotions.
\end{abstract}

Keywords: facial emotions, engagement, emotion detection

\section{INTRODUCTION}

Expressions are also used not only to express our feelings but also to provide essential communicative cues in social interaction, such as our degree of self-interest, continuous feedback signaling understanding of the information conveyed. Real-time system to recognize the basic emotions from a video. The system automatically detects frontal faces in the video stream and codes each frame concerning 6 dimensions: Neutral, anger, disgust, fear, sadness, surprise. The facial features from the video source are extracted and mapped with basic emotions. This can be used in any kind of environment. We use this system classroom environment to identify the involvement of students during lectures.

Revised Manuscript Received on April 30, 2020.

* Correspondence Author

Ashok kumar S*, Department of Information Technology, Sri Shakthi Institute of Engineering and Technology, Coimbatore, India. Email: sashokcs@gmail.com

Ragul R.N, Department of Information Technology, Sri Shakthi Institute of Engineering and Technology, Coimbatore, India. Email: ragulrnr7@gmail.com

Praveen Kumar S, Department of Information Technology, Sri Shakthi Institute of Engineering and Technology, Coimbatore, India. Email: praveensenthi03@gmail.com

Gokula krishnan D, Department of Information Technology, Sri Shakthi Institute of Engineering and Technology, Coimbatore, India. Email: gokulakrishnan1028@gmail.com

(c) The Authors. Published by Blue Eyes Intelligence Engineering and Sciences Publication (BEIESP). This is an open access article under the CC-BY-NC-NDlicense

(http://creativecommons.org/licenses/by-nc-nd/4.0/)
Body posture is two or even more muscle gestures or positions under the skin of the neck. Facial expressions are a visual and auditory mode of communication.

The important fact that feeling emotions are only one type of facial expression apart from others, such as listening, looking, shifting, or physiological activity. To show our emotional states and control the interactions we use facial expressions. In one of the six so-called essential emotions, the word "recognition of facial expression" also applies to the grouping of facial features: cheerful, bad, afraid, discomfort, shocked, upset, and neutral. By these expressions, we can examine the behavior of the student during the lecture. The involvement of students can be identified by their facial emotions. The face speaks more than words, it's better to convey messages. Non-verbal communication in the classroom is more important, as students express their situation or feelings by facial emotion or expressions. Face action units such as eyes, ears, nose, and front are markers of emotion. Here we examined whether the students 'emotion about comprehension is indicated through expressions of units of facial action. In this analysis, the key hypothesis of the first phase indicated that facial expression is the students in the classroom's commonly used one to one communication mode, which in effect helps lecturers recognize the students 'understanding. The second move indicated that facial expressions through the students 'action units help the lecturers recognize students' participation and comprehension during the lecture in the classroom. The third stage suggested that the expression of the student be substantially associated with their feelings, which in turn define their level of understanding. The study's significance has been analyzed statistically. Facial expression plays a significant role in defining the feelings and perceptions of students that are geographically dispersed as a group in the lecture period. Communication in a virtual group is similar to communication in a real classroom.

\section{LITERATURE SURVEY}

Validation of both the body language of the students can be used to realize their stage of attention. In a common atmosphere of the lecture space, instructors inform the training and often screen and involve the students in assessing their grasp and development. Two In this process, we used technical know-how interactive video-capture facial recognition on how to robotically realize the facial expressions of college students as a skill of inspecting their framework. [1].

The study of facial movements and comprehension of their emotional significance is a daunting task with wide applications. This immediately identifies human figures in images using algorithms from the neural community. 
That then locates and tests the characteristics of each facial expression student in the form room including pupils, lips, and jaw, etc..[2].

After the procedure of picture regularization, face emotion areas of a picture are stored in the database In this process, it assesses eight facial expression classification models for the mixtures of more than one photo of students in the college room.

These adjustments in texture and structure are rather special for different expressions [3]

Such procedures have proven difficult to prolong to multiple views, and have regularly been pretty fragile. Research in human techniques of face recognition, moreover, has proven that man or woman features and their immediate relationships contain an inadequate representation to account for the performance of grownup student face identification The established variable of the study was naming eight emotional facial expressions. The skill of naming thoughts was once defined as a verbal declaration via the concern of a corresponding emotional expression that seemed on the face expression of student character. The facial expressions are used to identify the six to eight of these had been regarded basic feelings regularly encountered in everyday life [4]

Much of the work in pc interest of Students face has situated on detecting university students such as the eyes, nose, mouth, and head outline, and defining a face model with the useful resource of the position, size, and relationships among these features. The communication of ideas fascinating It is that these expressions of emotion seem to differ (e.g., rage, hatred, panic, joy, sorrow, disappointment, and to a lesser extent disdain, humiliation, desire, discomfort, and disgrace) would possibly also moreover be biologically hard-wired, and are expressed the identical way by the ability of way of all person of all tradition. This contrasts with one of a variety of views that everyone facial expression is a product of social gaining understanding of and way of existence [5]

Students snapshots show up identical in any other case with the little variant in facial expression, facial details, pose, and many others. Outcomes of varying the variety of images per classification used in the training set from one to five for and also for the eight- the face of students [6]

The teacher may wish to have a full view of the classroom. Earlier, as an example, it is possible to decide how to classify the student to distinguish the pleasant students from them in difficulty; within the latter category, to differentiate the faculty students who applied several exercises that proved to be false from either the classes caught in the primary tasks; or to the center of attention on which workouts came resolutely be the foremost difficult. At any other point, the trainer may intend to get a glimpse of the student's actions, particularly in students of behavior, to learn unless the students applied the practice often, frequently or even more, if a person did anything else until going down. The instructor can, at last, be able to move from one view to the next. Their philosophy is to provide help for concentration purposes to the instructor. Visualizing devices, for example daily vision of the learning environment; vision of the end actions of a certain exercise; view of a specific student, can come from different factors of view. The program is designed to develop a range of visualization equipment and a computer for navigating through these additional devices. The trainer may be curious about understanding how lengthy college students want to operate an activity.[7,8]
At the quit of the locate out about it used to be regarded that the student expression like as happy-sad facial expressions at the level assembly the criteria, however, can also desire to no longer generalize them with continuing to be modeling and, likewise, learned tired-scared facial expressions then again should generalize them with facial expression. Social validity and maintenance had been no longer blanketed in the study.[9-11]

\section{DESIGN PROCESS}

The principal purpose of this lookup work is to classify the emotional expression of the student's face. As the initial task is to extract the full face area from the facial image, a survey on a variety of present research works to section the facial expression photographs are reviewed and discussed.

The established variable of the study was naming six emotional facial expressions. The skill of naming thoughts was once defined as a verbal declaration via the concern of a corresponding emotional expression that seemed on the face expression of student character. The facial expressions are used to identify the six of these had been regarded as basic feelings regularly encountered in everyday life

Among the most important elements for students, contact is the facial features. The face is responsible for not only thoughts or concepts but also feelings now speaking. This makes emotional interaction interesting is it almost seems as if several such expressions of emotion (e.g., furious, irritated, afraid, surprised) may be increasingly wired genetically; and are expressed identically by way of all peoples of all cultures.

The latter correlates with numerous views that each body language is a result of social awareness learning and the environment. Once was the predominant opinion that several such facial expressions of feeling derived from the evolution of the human race. Such expressions enabled the individual to live in shaping the story as it seemed necessary to behavioral animals such as humankind or apes to explain such implied in-between behaviors with the aid of the thoughts (trying to run away and hides, attacking in anger) so that they could escape the confrontation, risk, or allow approach, then on. Such emotional signals, however, are not immune to adjustment by social learning; various cultures evaluate extraordinary show policies to control their emotional expressions. Advances in upcoming apps will allow lookup of facial characteristics and amplify that fixes almost all of the remaining mandatory troubles.

The face of students automatically detects and maintains the student's progress like attendance and their expression in their lecture period or days. Therefore the attendance of the student can be made available by recognizing the face in the classroom.

Representation of the facial gestures of the students can be used to understand that level of focus. Teachers inform the lessons in a traditional classroom environment, and constantly expose and engage college students to understand their understanding and development. Despite the contemporary awareness of learning environments, the commitment of the diploma of interest in the on-line learning cycle has become crucial. For this analysis we utilized interactive easy-to-recognition video-capture science. 
Facial expression recognition is turning into more and more famous due to the fact of the need for human-machine interaction. The focus of facial expressions is used as one of a kind scholar can differ in the way that they exhibit their expressions. And even an image of the identical person in one expression can range in brightness, historical past and position.

\section{PROPOSED WORK}

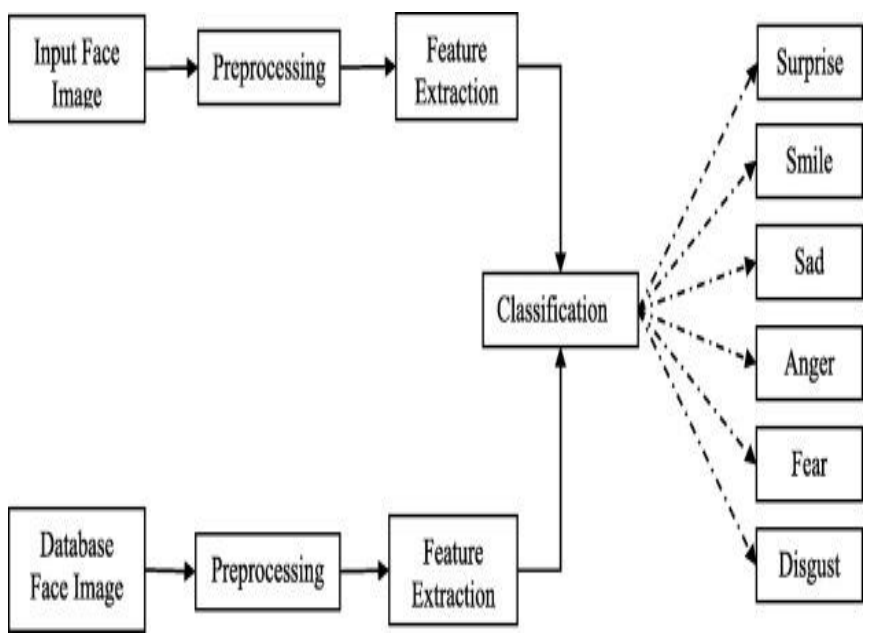

Fig 3.1: Face detection Flow Diagram

Face gestures that sign thoughts include muscle movements such as elevating your eyebrows, wrinkling your forehead, rolling your eyes, or curling your lip. Hence facial action tools such as pupils, mouth, eyebrow and lip are the markers of emotion. Here we examined whether the students 'emotions concerning comprehension are indicated through expressions of facial action units. In this approach, the use hypothesis of the first stage suggested that facial expression is the commonly used nonverbal mode of communication by the college students in the classroom which, in effect, is analogous to the actual communication, helps the lecturers find the students level. The second wave suggested that the facial expressions in the units of action help the lecturers fully understand the students 'participation and comprehension during the lecture. The third level indicated that the gestures of the student be substantially associated with their feelings, which in effect define their understanding level.

The study's importance was interpreted statistically. Face expression plays a critical role in defining the feelings and perceptions of students widely dispersed as classroom contact in a real classroom in college classrooms.

\section{A. Input Face Image:}

Most face detection systems try to extract a fraction of the complete face, thereby removing most of the background and other areas of somebody's head like hair that is not any longer vital for the face consciousness task. With static images, this can be frequently performed by employing jogging throughout the image. The face detection gadget then judges if a face is existing inner the window (Brunelli and Poggio, 1993). Unfortunately, with static photographs, there's a giant search area of feasible locations of a face in a picture
Face detection is taken input the photographs from digicam and output saved in region area and measurement of faces. The output of the face detection gadget can be a face recognition data, face monitoring, face authentication, face ex-press focus, and facial gesture recognition device. If the facial image with its frame measurement and place of frame, illumination or orientation can be normalized to continue face detection.

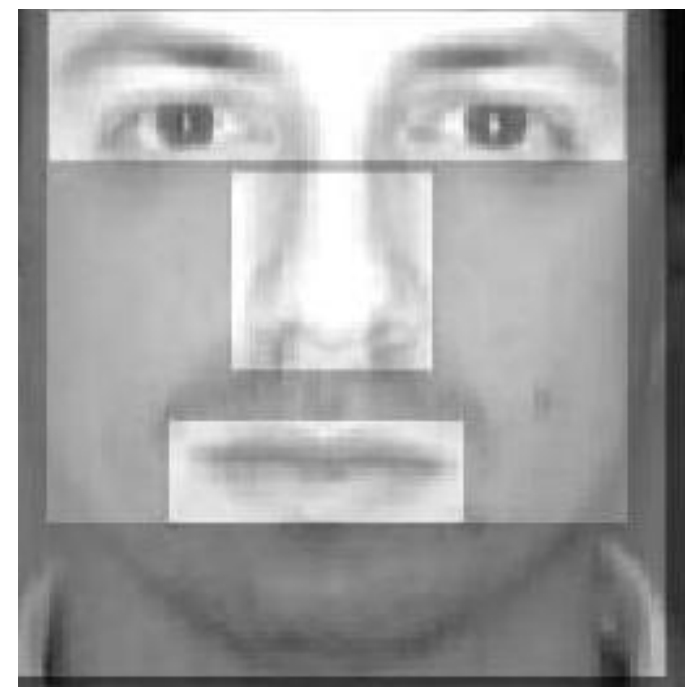

Fig 3.2: Face Recognition using Template matching

\section{B. Pre Processing}

The obtained data is usually messy and comes from special sources. To feed them to the ML model (or neural network), they have standardized and cleaned up. More often than not, pre-processing is used to habits steps that minimize the complexity and enlarge the accuracy of the applied algorithm. one can't write a single algorithm for every of the condition in which a photo is taken, thus, when a person collects a picture.

This step is to do away with inappropriate information of input photographs and beautify the detection ability of relevant information. Image preprocessing can immediately affect the extraction of points and the performance of expression classification. For quite a several reasons, pictures are often contaminated through some other signals. Some images can also nonetheless have complex backgrounds, e.g., light intensity, occlusion, and other interference factors, even if they are essentially free of noise. The established variable of the find out about used to be naming six emotional facial expressions. The skill of naming feelings was once described as a verbal declaration through the difficulty of a corresponding emotional expression that seemed on the face of the person opposite the problem after a tournament or scenario within four seconds. A literature overview was once carried out to decide the based variables of the study. Results of the literature evaluation about this subject showed that there were dozens of emotions and their corresponding facial expressions, however, six of these were considered simple emotions frequently encountered in everyday lifestyles

\section{FEATURE EXTRACTION:}

Feature extraction is an identity to more useful information 
from the student, e.g., name, section, and department. This used extract correction

. This approach is useful when an image stored in the database and match the correct person in the database

Facial feature extraction is the technique of extracting college students face element points like eyes, nose, mouth, and so on from human face image. Facial feature extraction is very tons necessary for the initialization of processing strategies like face tracking, facial expression recognition or face recognition.

\section{A. Classification Of Expression}

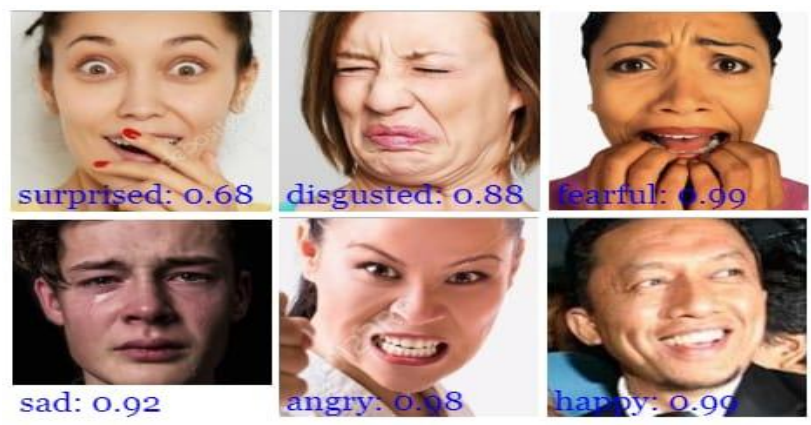

Fig 5.1: Classification of Expression

The device automatically acquired the college/school of students in the classroom whilst lecture taking a class. This approach used to locate the pupil mentally in the classroom

\section{B. Facial Detection}

Ability to become aware of the area of the face in any input photograph or frame. The output is the bounding container coordinates of the detected faces

A facial expression recognition machine is a computerized gadget which can analyze the facets of students face as an image or a stay video statistics set and pick out two students the facial expression into various classes. The expression of the face is an essential shape of mental and emotional state.

\section{Facial Recognition}

Compare multiple faces collectively to discover which faces belonging to an identifiable person. This is accomplished by evaluating face embedding vectors. Once a college student's face has been captured, the cropped photo will be relayed with an HTTP shape data request to the lower back end. This facial picture is then saved with the aid of the API, each on the nearby file system and in the detection log, appended with a student ID.

\section{Emotion Detection}

Classifying the emotion on the person/student face as angry, happy, surprise, neutral, sad and disgusted. In the total classroom, it can able to find the student expression and marking attendance and accurate of the result of each student Face detection is useful in understood students' expression in the classroom. Face Detection is carried out by a training dataset. This method implemented through a camera in the classroom. This system is also helpful for detecting and recognizing multiple faces in the classroom with acquired images. So it can reduce work for faculty and also concentrate on teaching class

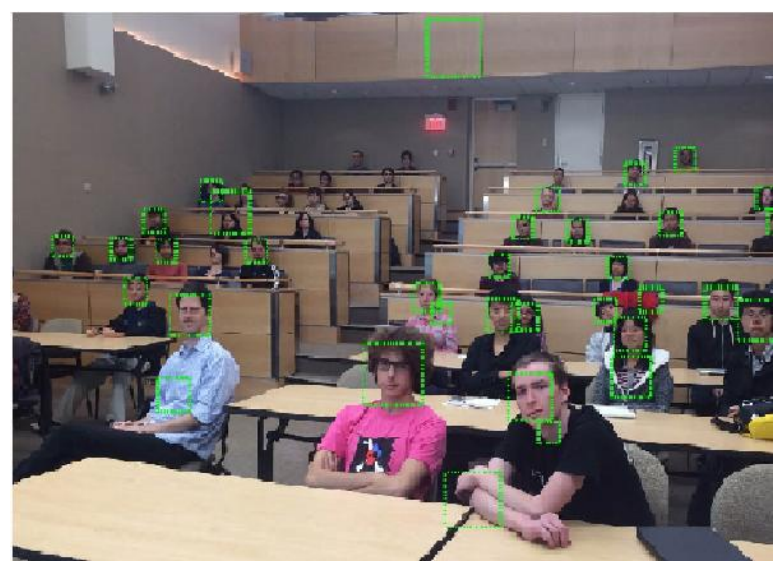

Fig 5.2: Real-time Facial Expression Detection Teacher-student Interaction performs an imperative position in any classroom surroundings. The effect due to communication of the face is so effective in interaction. Faces are prosperous in records about personal identity, and also about temper and intellectual state, being reachable home Window into the processes that control certain emotional responses. Studies also show since the most expressive way human beings convey feelings is through facial expressions. Facial expressions are used to find the individual's students or person inside feelings.

\section{RESULT AND DISCUSSION}

The accomplished training to implement makes use of the Convolution neural network-like function cascade detector to realize face as nicely as eyes and mouth. Detected faces are cropped, resized, and suggest subtracted, then PCA is performed. Also at some stage in training, eye and mouths are detected using Haar-like features, or using a Harris nook based strategy is Haar-like points fail.

\section{class A}

\section{Individual Expression}

\begin{tabular}{|l|l|l|l|}
\hline id & name & expression & \multicolumn{1}{|c|}{ Date } \\
\hline 1 & praveen & happy & Sat Mar 14 2020 17:42:41 \\
\hline 2 & gokul & sad & Sat Mar 14 2020 17:42:42 \\
\hline 3 & rahul & neutral & Sat Mar 14 2020 17:42:44 \\
\hline
\end{tabular}

\section{class A}

\section{Overall Expression}

\begin{tabular}{|l|l|l|}
\hline Expresssion & Percentage & \multicolumn{1}{|c|}{ Date } \\
\hline happy & $65 \%$ & Sat Mar 14 2020 17:45:41 \\
\hline neutral & $20 \%$ & Sat Mar 14 2020 17:45:41 \\
\hline sad & $10 \%$ & Sat Mar 14 2020 17:45:41 \\
\hline surprised & $5 \%$ & Sat Mar 14 2020 17:45:41 \\
\hline angry & $0 \%$ & Sat Mar 14 2020 17:45:41 \\
\hline disgusted & $0 \%$ & Sat Mar 14 2020 17:45:41 \\
\hline
\end{tabular}

Future work ought to entail improving the robustness of the classifiers by using including extra training snapshots from exceptional datasets, 
investigating more correct detection strategies that nevertheless keep computational efficiency, and thinking about the classification of extra nuanced and sophisticated expressions.

\section{CONCLUSION}

In summary, in the existing work seven expressions are each recognized and detected well in peripheral imaginative and prescient (i.e. happiness, shock, etc) whereas others are poorly identified and detected (i.e. angry and sad) whereas yet others change profile as a feature of undertaking (e.g. fear). Recent findings demonstrate that undertaking constraints structure the peripheral vision perception of speech and provide novel proof that identification and recognition of different underlying mechanisms are remembered. Lastly, our research stresses the importance of understanding the actual assignment and particular classes of expression used when assessing theoretical statements as to the value of speech processing for distinct basic categories of emotions.

\section{REFERENCES}

1. Chen, Hong-Ren. "Assessment of learners' attention to e-learning by monitoring facial expressions for computer network courses." Journal of Educational Computing Research 47.4 (2012): 371-385.

2. Bettadapura, V. (2012). Face expression recognition and analysis: the state of the art. arXiv preprint arXiv:1203.6722.

3. Tsai, Hung-Hsu, Yen-Shou Lai, and Yi-Cheng Zhang. "Using SVM to design facial expression recognition for shape and texture features." 2010 International Conference on Machine Learning and Cybernetics. Vol. 5. IEEE, 2010.

4. Turk, M., \& Pentland, A. (1991, January). Face recognition using eigenfaces. In Proceedings. 1991 IEEE computer society conference on computer vision and pattern recognition (pp. 586-587).

5. Turk, Matthew, and Alex Pentland. "Face recognition using eigenfaces." In Proceedings. 1991 IEEE computer society conference on computer vision and pattern recognition, pp. 586-587. 1991.

6. Lawrence, Steve, et al. "Face recognition: A convolutional neural-network approach." IEEE transactions on neural networks 8.1 (1997): 98-113

7. France, L., Heraud, J. M., Marty, J. C., Carron, T., \& Heili, J. (2006, July). Monitoring virtual classroom: Visualization techniques to observe student activities in an e-learning system. In Sixth IEEE International Conference on Advanced Learning Technologies (ICALT'06) (pp. 716-720). IEEE.

8. France, Laure, et al. "Monitoring virtual classroom: Visualization techniques to observe student activities in an e-learning system." Sixth IEEE International Conference on Advanced Learning Technologies (ICALT'06). IEEE, 2006

9. Leipheimer, Kristin, and Janine Stichter. "Teaching facial expressions of emotion." Beyond Behavior 21.1 (2012): 20-28

10. Leipheimer, Kristin, and Janine Stichter. "Teaching facial expressions of emotion." Beyond Behavior 21.1 (2012): 20-28.

11. Leipheimer, Kristin, and Janine Stichter. "Teaching facial expressions of emotion." Beyond Behavior 21, no. 1 (2012): 20-28.

12. Niedenthal, Paula M., et al. "Emotional state and the detection of the change in facial expression of emotion." European journal of social psychology 30.2 (2000): 211-222.

13. Niedenthal, P. M., Halberstadt, J. B., Margolin, J., \& Innes-Ker, Å. H. (2000). Emotional state and the detection of the change in facial expression of emotion. European journal of social psychology, 30(2), 211-222.

\section{AUTHORS PROFILE}

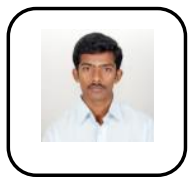

Mr. S Ashok kumar, currently working as Assistan Professor in the Department of Information Technology, Sri Shakthi Institute of Engineering and Technology, Coimbatore. And, Completed Post Graduation in Computer Science and Engineering from Anna University of Technology, Coimbatore, Under Graduation in Information Technology from Sona College of Technology, Salem and have 9 years of teaching experience. Life member of ISTE, and IE.

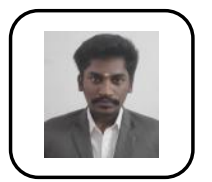

R. N. Ragul, currently studying Under Graduation in the Department of Information Technology, Sri Shakthi Institute of Engineering and Technology, Coimbatore.

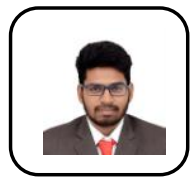

S Praveen Kumar, currently studying Under Graduation in the Department of Information Technology, Sri Shakthi Institute of Engineering and Technology, Coimbatore.

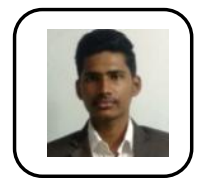

D. GokulaKrishnan, currently studying Under Graduation in the Department of Information Technology, Sri Shakthi Institute of Engineering and Technology, Coimbatore. 